\title{
Nontarget organism effects tests on eCry3.1Ab and their application to the ecological risk assessment for cultivation of Event 5307 maize
}

\author{
Andrea Burns $\cdot$ Alan Raybould
}

Received: 30 August 2013/Accepted: 16 December 2013/Published online: 10 January 2014

(C) The Author(s) 2014. This article is published with open access at Springerlink.com

\begin{abstract}
Event 5307 transgenic maize produces the novel insecticidal protein eCry3.1Ab, which is active against certain coleopteran pests such as Western corn rootworm (Diabrotica virgifera virgifera). Laboratory tests with representative nontarget organisms (NTOs) were conducted to test the hypothesis of no adverse ecological effects of cultivating Event 5307 maize. Estimates of environmental eCry3.1Ab concentrations for each NTO were calculated from the concentrations of eCry3.1Ab produced by 5307 maize in relevant plant tissues. Nontarget organisms were exposed to diets containing eCry3.1 Ab or diets comprising Event 5307 maize tissue and evaluated for effects compared to control groups. No statistically significant differences in survival were observed between the control group and the group exposed to eCry3.1Ab in any organism tested. Measured eCry3.1Ab concentrations in the laboratory studies were equal to or greater than the most conservative estimates of environmental exposure. The laboratory studies corroborate the hypothesis of negligible ecological risk from the cultivation of 5307 maize.
\end{abstract}

A. Burns $(\square)$

Syngenta Crop Protection, LLC, 3054 East Cornwallis Road, Post Office Box 12257, Research Triangle Park, NC 27709, USA

e-mail: andrea.nelson@syngenta.com

\section{A. Raybould}

Jealott's Hill International Research Centre, Syngenta,

Bracknell, Berkshire RG22 4JN, UK
Keywords Ecological risk assessment . Hypothesis testing $\cdot$ Hazard quotient $\cdot$ Bt crops

\section{Introduction}

Syngenta's Event 5307 transgenic maize (Agrisure Duracade $^{\mathrm{TM}}$ corn) produces the novel insecticidal protein eCry3.1 $\mathrm{Ab}$, an engineered chimera of Cry $1 \mathrm{Ab}$ and modified Cry $3 \mathrm{~A}$, which are both derived from Bacillus thuringiensis. In screening assays conducted with leaf tissue of 5307 maize, or with microbially produced eCry3.1 Ab, no activity was observed against any Lepidopteran tested (Agrotis ipsilon-black cutworm; Helicoverpa zea-corn earworm; Spodoptera frugiperda-fall armyworm; Heliothis virescenstobacco budworm; Ostrinia nubilalis-European corn borer; and Pectinophora gossypiella - pink bollworm). Activity was observed only against certain coleopteran pests in the Chrysomelidae, such as the Western corn rootworm (Diabrotica virgifera virgifera) (Vlachos and Huber 2011).

Syngenta's Event MIR604 transgenic maize, which produces a modified Cry3A (mCry3A), also has resistance to Western corn rootworm. Walters et al. (2010) generated data corroborating the hypothesis that eCry3.1 Ab can interact with different binding sites than the activated form of mCry $3 \mathrm{~A}$ in the gut brush border membrane of the Western corn 
rootworm. Therefore, mCry $3 \mathrm{~A}$ and eCry3.1Ab may have different modes of action, which is an advantageous strategy for insect resistance management (Bravo and Soberón 2008).

Prior to approving transgenic crops for commercial cultivation, regulatory agencies assess the environmental risk of cultivating the genetically modified (GM) crop. Laboratory studies are conducted to expose representative nontarget organisms (NTOs) directly to the GM plant material or to concentrations of the insecticidal protein that exceed the concentrations to which they will be potentially exposed in the field. These laboratory studies are conducted to test the hypothesis of no adverse ecological effects from cultivation of the crop. Following a tiered testing approach, if direct exposure to elevated concentrations of the insecticidal protein indicates no adverse effects, then no further testing is required to conclude negligible ecological risk from exposure to protein concentrations resulting from cultivation of the crop. If adverse effects are observed, additional laboratory testing where organisms are exposed to more realistic concentrations of the purified $\mathrm{Bt}$ protein or plant material may be conducted if further characterisation of risk is deemed necessary for decision-making. Meta-analysis has indicated that laboratory studies conducted to assess the effects of Bt crops are predictive of, if not more conservative than, field level effects (Duan et al. 2010).

This paper presents estimates of environmental concentrations of eCry3.1Ab via the cultivation of Event 5307 maize and of no-observed-adverse-effectconcentrations from NTO laboratory studies. This information is combined to assess the ecological risk of cultivating Event 5307 maize.

\section{Materials and methods}

eCry3.1Ab test substances

During the development of Event 5307 maize several NTO effects tests were conducted with plant or microbially produced test substances (Tables 1, 2) containing eCry3.1Ab. To achieve higher eCry3.1Ab test concentrations than are possible using plantderived test substances, test substance was produced by expressing the ecry3.1Ab gene in Escherichia coli and purifying the resulting eCry3.1Ab. An evaluation of equivalence (Raybould et al. 2013) comprising comparisons of apparent molecular weight, antibody cross-reactivity, bioactivity against a sensitive insect species, and glycosylation status concluded that effects tests using the microbial produced test substance will conservatively predict the effects of eCry3.1Ab in Event 5307 maize (Vlachos and Huber 2011).

\section{Effects of eCry3.1Ab on surrogate non-target organisms}

Tables 1 and 2 summarize the species and life-history stage tested, the functional groups represented, the routes of test organism exposure to eCry3.1Ab, the sample size, and the duration and physical conditions of the study. Species were chosen to comply with regulatory requirements in countries where cultivation approvals for Event 5307 maize may be sought. The species also fulfil many of the criteria for choosing laboratory test organisms to provide reliable information for ecological risk assessment (Romeis et al. 2013).

Table 1 Species used in effects tests of eCry3.1Ab

\begin{tabular}{lllll}
\hline Test species & Common name & Order: family & Life stage & Group represented \\
\hline Coleomegilla maculata & Pink-spotted ladybird beetle & Coleoptera: Coccinellidae & Larva & Foliar non-target arthropods \\
Orius laevigatus & Flower bug & Hemiptera: Anthocoridae & Nymph & Foliar non-target arthropods \\
Aleochara bilineata & Rove beetle & Coleoptera: Staphylinidae & Adult & Soil-dwelling invertebrates \\
Poecilus cupreus & Carabid beetle & Coleoptera: Carabidae & Larva & Soil-dwelling invertebrates \\
Eisenia fetida & Earthworm & Haplotaxida: Lumbricidae & Adult & Soil-dwelling invertebrates \\
Apis mellifera & Honey bee & Hymenoptera: Apidae & Brood & Pollinators \\
Colinus virginianus & Bobwhite quail & Galliformes: Phasianidae & Juvenile & Wild birds \\
Mus musculus & Mouse & Rodentia: Muridae & Young adult & Wild mammals \\
Gammarus fasciatus & Freshwater shrimp & Amphipoda: Gammaridae & Adult & Freshwater invertebrates \\
Ictalurus punctatus & Channel catfish & Siluriformes: Ictaluridae & Juvenile & Fish (farmed and wild) \\
\hline
\end{tabular}




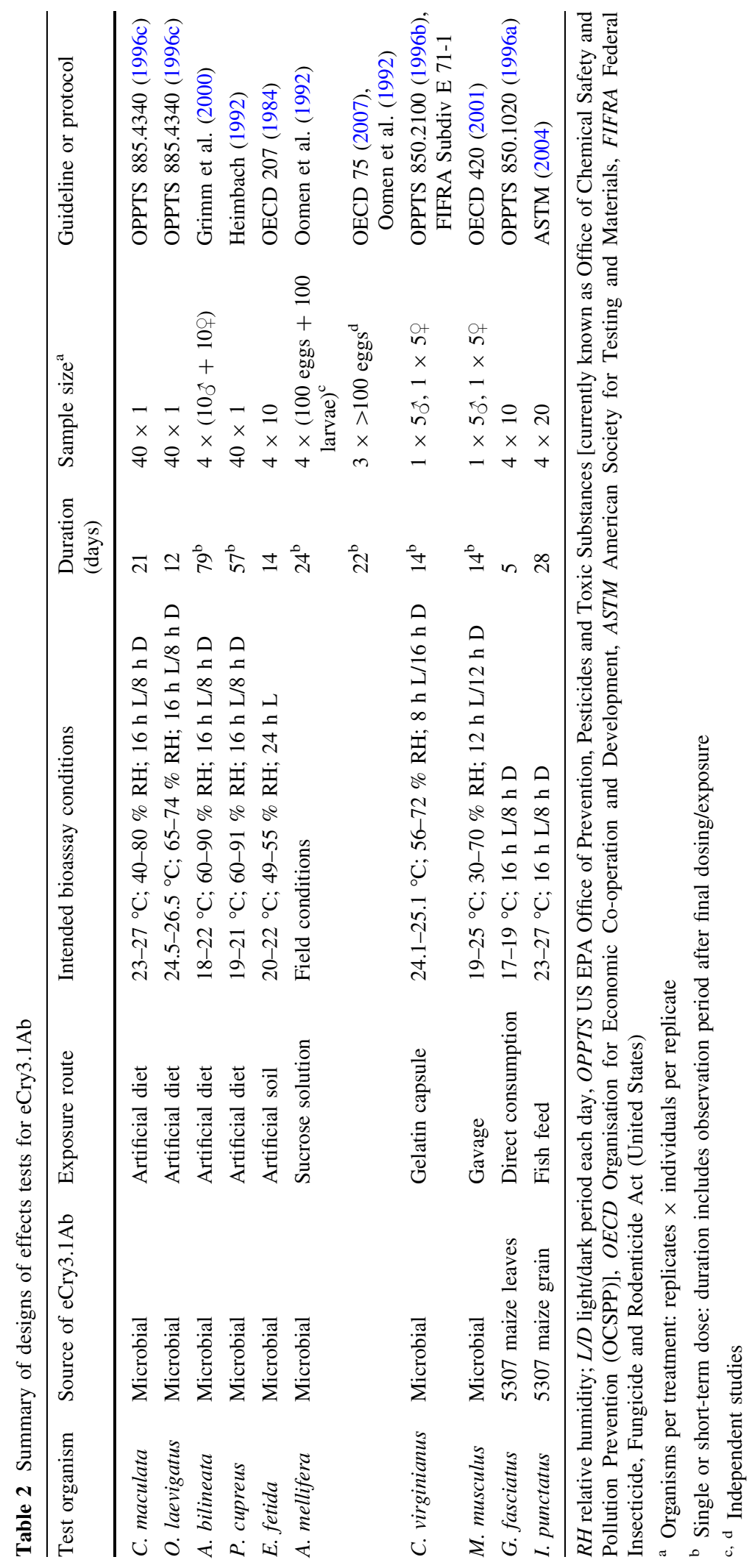


Each study followed a relevant guideline or protocol from organizations such as the Organization for Economic Co-operation and Development (OECD) and the United States Environmental Protection Agency (US EPA) (Table 2). These guidelines specify sample sizes, test endpoints and criteria to determine the validity of the study. All studies except for the bees met validity criteria for positive and negative control mortality. The study guideline for bees by Oomen et al. (1992) indicates that control mortality should not be, "generally above $15 \%$." While the control mortality exceeded this minimum acceptable level, the studies were still informative for evaluating the effects of eCry3.1 Ab on brood development because the test concentration that the bees were exposed to was much greater than the concentration produced in 5307 maize pollen. The data were more robust the closer the control mortality was to $15 \%$. In some studies, physical conditions sporadically varied outside the set limits; however, in no case was the study deemed to have breached the validity criteria of the protocol.

In each study, a negative control group was exposed to the same diet, vehicle, or test solution as the test group without the addition of eCry3.1Ab. In some instances a positive control treatment was included. In each case the reference substance used to produce the positive control treatment is expected to have effects on the organism being tested. The positive control treatment provides confirmation that the organism was exposed to the diet, vehicle, or test solution.

The composition of the diets used to expose test organisms are summarised here. Full details of the diets and the physical attributes of the test systems are available on request. The Coleomegilla maculata diet was a mixture of $50 \%$ eggs of the moth Ephestia kuehniella and $50 \%$ bee pollen collected from various flowers. The diet for the Orius laevigatus, Aleochara bilineata and Poecilis cupreus studies comprised a blend of roughly $46.5 \%$ minced beef, $46.5 \%$ lamb's liver, $4.6 \%$ yeast flakes and $2.4 \%$ clear honey by weight. The beef and liver were cooked in an oven at $72{ }^{\circ} \mathrm{C}$ for $30 \mathrm{~min}$ and in an $800 \mathrm{~W}$ microwave oven on full power for $5 \mathrm{~min}$ to denature enzymes that might degrade the eCry3.1Ab. These components were liquidised and blended with a mixture comprising $62.3 \%$ whole beaten egg, $9.3 \%$ sucrose, $0.4 \%$ Nipagin M preservative and $28 \%$ purified water by weight. The finished diet comprised $57 \%$ of the meat and liver mixture and $43 \%$ of the egg and sugar mixture by weight.

Honeybees were exposed to eCry3.1Ab via a $50 \%$ weight by volume sucrose solution. The test diet in the Gammarus fasciatus study was leaf discs of 5307 maize that had been soaked for 4 days in the same water as used in the test system. A control group was exposed to leaf discs from non-transgenic maize nearisogenic to 5307 maize. Bobwhite quail were exposed via gelatin capsules containing eCry3.1 $\mathrm{Ab}$, and mice were exposed to eCry3.1Ab via gavage using carboxymethylcellulose as the dosing vehicle.

Catfish were exposed to eCry3.1Ab via a standard catfish diet prepared from $41 \%$ grain derived from 5307 maize. The control group was fed a similar diet prepared from grain derived from non-transgenic maize near isogenic to 5307 maize. Finally, earthworms were exposed via an artificial soil comprising $10 \%$ sphagnum peat, $20 \%$ kaolinite clay, $69.8 \%$ quartz sand and $0.2 \%$ calcium carbonate by weight.

Table 3 lists the concentrations of eCry3.1Ab to which the organisms were exposed in the effects tests and the endpoints measured. Several considerations were used to select the test concentrations, including regulatory requirements, the predicted exposures in the field, the desire to model worst-case exposures and apply conservative assumptions and the practical limitations of the test system. The nominal eCry3.1Ab test concentration is given when the test substance was supplied in a single dose by gelatin capsule, by gavage or in aqueous solution prepared fresh daily for the duration of exposure. When plant material was used for exposure, the percentage incorporation of plant material in the diet is indicated.

In studies where a microbially produced test substance was repeatedly supplied via an artificial diet, aliquots of treated diet were stored frozen and freshly thawed aliquots were supplied daily or every other day to the test species. Remaining aliquots were analyzed at the end of the in-life phase of the study to confirm the stability of eCry3.1Ab in the diet. The concentration of bioactive eCry3.1 Ab in the diet was inferred from a weight of evidence based on the following assays: enzyme-linked immunosorbent assay (ELISA) to measure eCry3.1Ab concentration; western blotting to measure intactness of eCry3.1Ab; and a sensitive insect bioassay to confirm bioactivity. See Table 3 for details of diets that were analyzed. 


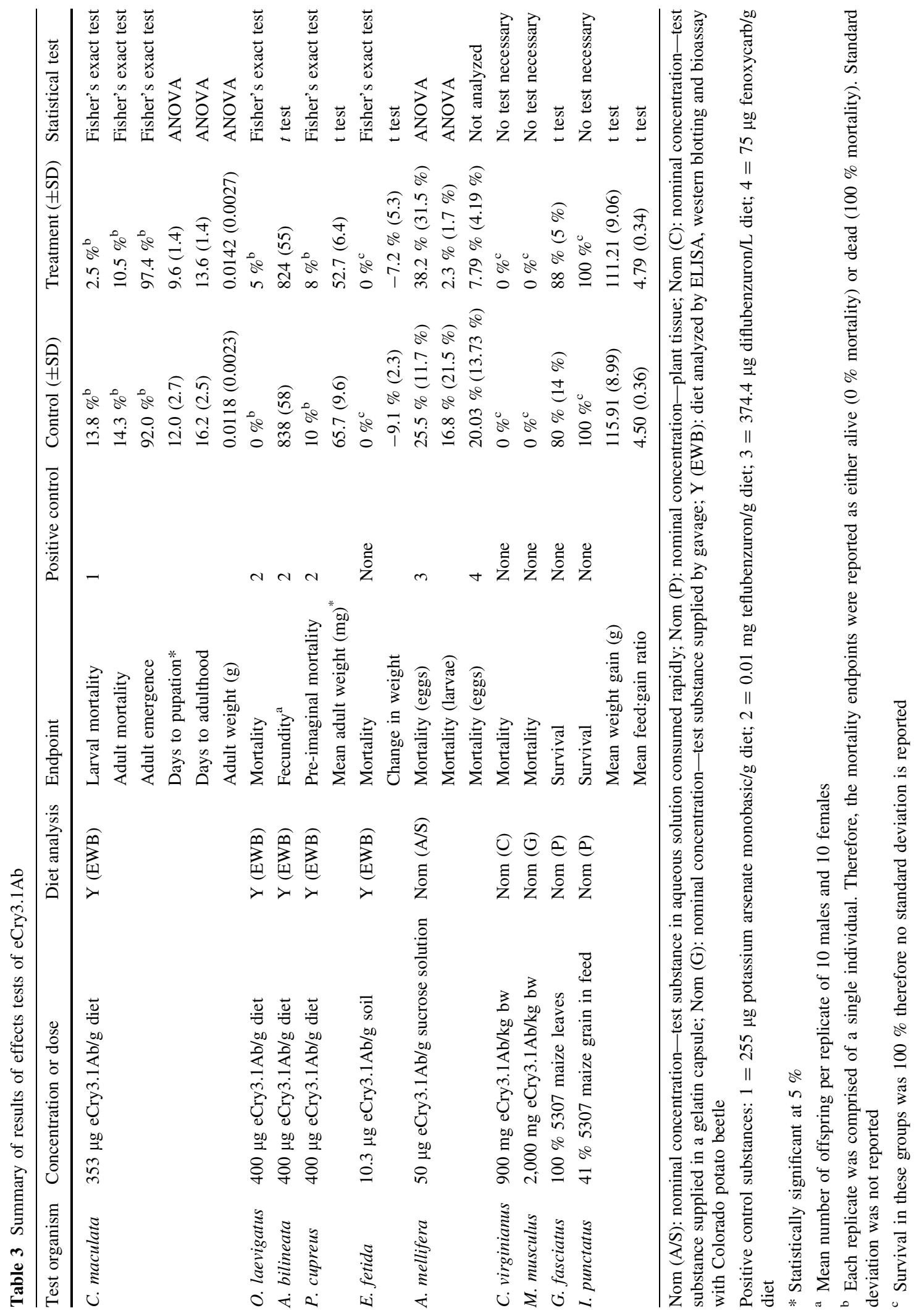


Concentrations of eCry3.1Ab in Event 5307 maize

Event 5307 maize was grown using standard local agronomic practices at three field locations in Illinois and one location in Minnesota. Concentrations of eCry3.1 Ab in various tissues at several developmental stages were determined by ELISA and corrected for extraction efficiency (e.g., Nguyen and Jehle 2009). Tissues from nontransgenic, near-isogenic control plants were collected concurrently and analyzed in a similar manner to test for lack of eCry3.1Ab or interference from background substances.

Estimated environmental concentrations

of eCry3.1 Ab from Event 5307 maize

Predicted exposures of non-target organisms to insecticidal proteins from cultivation of a transgenic crop are known as estimated environmental concentrations (EECs). For NTOs potentially exposed to eCry3.1Ab, EECs were estimated using data on the concentrations of eCry3.1Ab in relevant Event 5307 maize tissues. In order to calculate the EECs in a highly conservative manner, the highest mean eCry3.1Ab concentration reported for the relevant tissue at any single field trial location was used.

The diet of terrestrial invertebrates was assumed to be $100 \%$ of the relevant Event 5307 maize tissue; thus, for these organisms, the EEC is equal to the highest mean concentration of eCry3.1Ab in that tissue measured at any single field trial location. These EECs are extremely conservative and present a worstcase exposure scenario because it is highly unlikely that the diet of these organisms is comprised solely of maize tissue (e.g., Raybould et al. 2007; Raybould and Vlachos 2011). Data on the environmental fate of eCry3.1Ab further confirm that the exposures tested were highly conservative. It has been demonstrated that eCry3.1Ab loses bioactivity in soil within 14 days; therefore, it is unlikely to persist or accumulate in the environment and result in longer-term exposures (Vlachos and Huber 2011).

For birds and wild mammals, exposure was estimated as a daily dietary dose (DDD), not as a concentration. DDDs were calculated from estimates of the food intake rates and body weights of seedeating birds and rodents (Crocker et al. 2002) as described by Raybould et al. (2007). The worst-case (most highly conservative) DDDs for birds and mammals were calculated as $1.94 \mu \mathrm{g}$ eCry3.1Ab/g body weight and $1.82 \mu \mathrm{g}$ eCry $3.1 \mathrm{Ab} / \mathrm{g}$ body weight, respectively.

For farmed fish, the worst-case EEC was calculated assuming that fish feed is comprised of $30 \%$ maize (NRC 1983) and that all the maize in the diet is Event 5307 grain. The worst-case exposure scenario also assumes that no eCry3.1 $\mathrm{Ab}$ activity is lost during the preparation of the feed; this is unlikely as fish feed is heat-treated during preparation and eCry3.1Ab has been shown to lose biological activity after heat treatment (Vlachos and Huber 2011).

For aquatic invertebrates, potential exposure via pollen or plant debris transfer into waterways and runoff was considered. Dietary exposure to plant debris was considered to be the most conservative theoretical scenario, and therefore the worst-case EEC was taken as the highest mean concentration of eCry3.1Ab in leaves of Event 5307 maize.

Risks to non-target organisms

A conservative formulation of the hypothesis that eCry3.1 Ab in Event 5307 maize will not harm NTOs is that no NTO will be exposed to a concentration or dose of eCry3.1 Ab greater than the highest concentration or dose of eCry3.1Ab that has no observed adverse effect (i.e., the no observed adverse effect concentration or level; NOAEC or NOAEL, respectively). The hypothesis of no adverse effects on NTOs from exposure to eCry3.1Ab in the field was tested by comparing the NOAEC obtained from the laboratory effect studies with the EEC estimates derived as described above. If the ratio EEC/NOAEC (the hazard quotient; HQ) is no greater than 1 for all groups of NTOs, the risks posed by exposure to eCry3.1Ab from cultivation of Event 5307 may be considered negligible (Raybould et al. 2007; Romeis et al. 2008; Raybould and Vlachos 2011).

\section{Results}

Estimated environmental concentrations of eCry3.1 $\mathrm{Ab}$

The fresh-weight concentrations of eCry3.1Ab in Event 5307 maize tissues are summarized in Table 4. eCry3.1Ab was detected in all tissues analyzed. For 
each tissue type, the highest mean eCry3.1Ab concentration at a single trial location at any developmental stage is reported. This concentration is used in the calculation of worst-case EECs.

Worst-case EECs of eCry3.1Ab for various NTO groups that could potentially be exposed via cultivation of Event 5307 maize are given in Table 5.

Table 4 Highest mean fresh weight concentrations of eCry3.1 Ab in 5307 plants grown at 3 locations in Illinois and 1 location in Minnesota

\begin{tabular}{lcl}
\hline Tissue $^{\mathrm{a}}$ & $\begin{array}{l}\text { Highest mean at any } \\
\text { site }(\mu \mathrm{g} \text { eCry3.1Ab/g } \\
\text { fresh wt.) }\end{array}$ & $\begin{array}{l}\text { Growth } \\
\text { stage }\end{array}$ \\
\hline Leaf & 51.74 & Maturity \\
Kernel & 5.53 & Maturity \\
Root & 6.48 & Whorl \\
Pollen & $0.22^{\mathrm{b}}$ & Anthesis \\
Whole plant & 18.62 & Whorl \\
Whole plant & 4.89 & Senescence \\
\hline
\end{tabular}

${ }^{\mathrm{a}}$ For leaf, kernel, root, pollen and whole plant, $\mathrm{N}=5$ plants for each location

b Pollen samples collected from Illinois and Minnesota showed eCry3.1Ab concentrations either equal to or less than the LOQ $(0.1 \mu \mathrm{g}$ eCry3.1Ab/g dry weight pollen). Pollen collected from 5307 maize plants grown in Brazil showed higher levels of eCry3.1Ab in pollen than previously measured from the locations in Illinois and Minnesota. To be conservative the highest mean concentration measured in the Brazilian field trial (1 location) has been used here to calculate the EEC
eCry3.1Ab effects tests

Results of the effects tests with eCry3.1Ab are summarized in Table 3. In 8 of the 10 species tested, there were no statistically significant differences between the organisms exposed to eCry3.1Ab and those in the negative control group among any of the various endpoints assessed, including survival, weight, weight gain, fecundity, or development time. Among the Coleomegilla maculata exposed to microbially produced eCry3.1 Ab there was no statistically significant difference in pupal mortality, adult mortality, adult emergence, number of days to adult emergence or mean adult beetle weight; however, there was a statistically significant difference in number of days to pupation. The individuals exposed to eCry3.1 Ab reached pupation more quickly than the control group. This difference is not considered adverse and no statistical difference was reported in the subsequent measurement of the number of days to adult emergence. Among the P. cupreus larvae exposed to microbially produced eCry3.1 $\mathrm{Ab}$ there was no statistically significant difference in mortality; however, there was a statistically significant difference in emergent beetle weight of the eCry3.1Abtreated group when compared to the controls. On average the individuals exposed to eCry3.1Ab weighed $20 \%$ less than the controls.

For the $P$. cupreus study, therefore, the NOAEC is less than $400 \mu \mathrm{g}$ eCry3.1Ab/g diet based on the

Table 5 Summary of estimated environmental concentrations (EECs) of eCry3.1Ab via 5307 maize

\begin{tabular}{|c|c|c|}
\hline \multirow[t]{2}{*}{ NTO group } & \multicolumn{2}{|l|}{ Worst case exposure to eCry $3.1 \mathrm{Ab}$} \\
\hline & Route & Concentration or dose \\
\hline Foliar non-target arthropods & Diet $100 \% 5307$ maize leaves & $51.74 \mu \mathrm{g}$ eCry3.1Ab/g leaves \\
\hline Soil-dwelling invertebrates & Diet $100 \% 5307$ maize roots & $6.48 \mu \mathrm{g}$ eCry $3.1 \mathrm{Ab} / \mathrm{g}$ roots \\
\hline Pollinators & Diet $100 \% 5307$ maize pollen & $0.22 \mu \mathrm{g}$ eCry $3.1 \mathrm{Ab} / \mathrm{g}$ pollen \\
\hline Wild birds & Diet $100 \% 5307$ maize kernels & $\begin{array}{l}5.53 \mu \mathrm{g} \text { eCry3.1Ab/g kernels } \\
1.94 \mu \mathrm{g} \text { eCry } 3.1 \mathrm{Ab} / \mathrm{g} \text { body weight }\end{array}$ \\
\hline Wild mammals & Diet $100 \% 5307$ maize kernels & $\begin{array}{l}5.53 \mu \mathrm{g} \text { eCry } 3.1 \mathrm{Ab} / \mathrm{g} \text { kernels } \\
1.82 \mu \mathrm{g} \text { eCry } 3.1 \mathrm{Ab} / \mathrm{g} \text { body weight }\end{array}$ \\
\hline Freshwater invertebrates & Diet $100 \% 5307$ maize & $\begin{array}{l}\text { Concentration of eCry3.1Ab in Event } 5307 \text { maize leaf discs } \\
\text { collected from greenhouse grown plants during the } \\
\text { vegetative stage of development (not quantified) }\end{array}$ \\
\hline Fish & $100 \% 5307$ maize grain & $30 \% 5307$ maize grain incorporated in fish feed \\
\hline
\end{tabular}


Table 6 Hazard quotients for organisms exposed to eCry3.1Ab via cultivation of 5307 maize

\begin{tabular}{|c|c|c|c|c|}
\hline Test species & Functional group & $\mathrm{EEC}$ & NOAEC/NOAEL & Worst-case HQ \\
\hline C. maculata & Foliar non-target arthropods & $51.74 \mu \mathrm{g}$ eCry3.1Ab/g diet & $353 \mu \mathrm{g}$ eCry $3.1 \mathrm{Ab} / \mathrm{g}$ diet & $\leq 0.147$ \\
\hline O. laevigatus & Foliar non-target arthropods & $51.74 \mu \mathrm{g}$ eCry3.1Ab/g diet & $400 \mu \mathrm{g}$ eCry $3.1 \mathrm{Ab} / \mathrm{g}$ diet & $\leq 0.130$ \\
\hline A. bilineata & Soil-dwelling invertebrates & $6.48 \mu \mathrm{g}$ eCry $3.1 \mathrm{Ab} / \mathrm{g}$ diet & $400 \mu \mathrm{g}$ eCry $3.1 \mathrm{Ab} / \mathrm{g}$ diet & $\leq 0.016$ \\
\hline P. cupreus & Soil-dwelling invertebrates & $6.48 \mu \mathrm{g}$ eCry $3.1 \mathrm{Ab} / \mathrm{g}$ diet & $\begin{array}{l}400 \mu \mathrm{g} \text { eCry } 3.1 \mathrm{Ab} / \mathrm{g} \text { diet } \\
<400 \mu \mathrm{g} \text { eCry3.1Ab/g diet }\end{array}$ & $\begin{array}{l}\leq 0.016 \text { (mortality) } \\
>0.016 \text { (development) }\end{array}$ \\
\hline E. fetida & Soil-dwelling invertebrates & $6.48 \mu \mathrm{g}$ eCry3.1Ab/g diet & $10.3 \mu \mathrm{g}$ eCry $3.1 \mathrm{Ab} / \mathrm{g}$ diet & $\leq 0.63$ \\
\hline A. mellifera & Pollinators & $0.22 \mu \mathrm{g}$ eCry $3.1 \mathrm{Ab} / \mathrm{g}$ diet & $50 \mu \mathrm{g}$ eCry $3.1 \mathrm{Ab} / \mathrm{g}$ diet & $\leq 0.004$ \\
\hline C. virginianus & Wild birds & $1.94 \mu \mathrm{g}$ eCry $3.1 \mathrm{Ab} / \mathrm{g}$ bw & $900 \mu \mathrm{g}$ eCry3.1Ab/g bw & $\leq 0.002$ \\
\hline M. musculus & Wild mammals & $1.82 \mu \mathrm{g}$ eCry $3.1 \mathrm{Ab} / \mathrm{g}$ bw & $2,000 \mu \mathrm{g}$ eCry $3.1 \mathrm{Ab} / \mathrm{g}$ bw & $\leq 0.0009$ \\
\hline G. fasciatus & Freshwater invertebrates & $100 \% 5307$ maize leaves & $100 \% 5307$ maize leaves & $\leq 1$ \\
\hline I. punctatus & Fish (farmed and wild) & $100 \% 5307$ maize grain & $41 \% 5307$ maize grain & $\leq 0.7$ \\
\hline
\end{tabular}

growth endpoint, or $400 \mu \mathrm{g}$ eCry3.1Ab/g diet based on the mortality endpoint.

Hazard quotients (HQ)

Table 6 lists the HQs for various functional groups. The HQ was calculated by dividing the worst-case EEC from Table 5 by the concentration or dose of eCry3.1Ab in the effects studies on species from the relevant functional group (Table 3); in all cases apart from the growth endpoint for $P$. cupreus, these values represent NOAECs or NOAELs as no effects were seen.

Using worst-case EECs, most of the HQs are well below 1. The exception is the G. fasciatus (freshwater invertebrate) which has a worst-case HQ of $\leq 1$. $G$. fasciatus was provided a diet comprised of only Event 5307 maize leaf discs therefore the exposure represents at least $1 \times$ the worst case EEC. It is unlikely that G. fasciatus would consume a diet comprised solely of fresh Event 5307 maize leaves. The Event 5307 maize leaf discs to which the G. fasciatus were exposed were collected from Event 5307 plants at the vegetative stage of development. It has been observed that input of crop by-products into waterways is highest several months after field harvest (Jensen et al. 2010). Therefore, post-harvest plant debris will most likely be derived from senescent plant material. For all of the developmental stages from which Event 5307 plant material was analyzed, the mean concentration of eCry3.1Ab was the lowest in senescent tissues. Aging of $\mathrm{Bt}$ maize tissue is associated with rapid loss of activity of the insecticidal proteins as measured by sensitive insect bioassay (Jensen et al. 2010). Therefore, because $G$. fasciatus were exposed to a diet comprised solely of Event 5307 maize leaf discs collected from the vegetative stage of plant development this represents at least $1 \mathrm{X}$ the worst-case exposure scenario.

\section{Discussion}

Apart from $P$. cupreus, no statistically significant adverse effects were observed following exposure to eCry3.1Ab, and worst-case HQs were well below 1 for most organisms. The general lack of adverse effects in studies in which representative indicator organisms were exposed to concentrations of eCry3.1Ab in excess of very conservative estimates of potential exposure corroborates the hypothesis that exposure to eCry3.1Ab via cultivation of Event 5307 maize will have little or no adverse effects on all NTOs, not only biological control organisms. Hence, the ecological risks from cultivating 5307 maize are negligible.

Gammarus fasciatus and Ictalurus punctatus tests involved exposure of the test group to plant material or a diet comprised of Event 5307 plant material. Studies where test groups are exposed to plant material can present a worst-case scenario by maximizing the percentage of Event 5307 plant material in the diet or collection of plant material from a particular plant developmental stage with high transgenic protein concentrations. To maximize exposure in the $G$. fasciatus study, the diet was comprised solely of Event 5307 leaf discs from the developmental stage 
with the reported highest level of eCry3.1Ab; in the field, aquatic invertebrates are likely to be exposed to aged Bt crop residues that have lost bioactivity (Jensen et al. 2010). For the I. punctatus study, exposure to Event 5307 maize grain was maximized by incorporating a high percentage of maize grain into the diet and all of the grain was derived from Event 5307 maize. The measured concentration of eCry3.1Ab in soil from the study with Eisenia fetida was less than nominal due to known difficulties with extracting Cry proteins from soil matrices (e.g. Palm et al. 1994).

For $P$. cupreus, HQs based on mortality were well below 1 , even for the extremely conservative worstcase exposure scenario; however, on average $P$. cupreus exposed to eCry3.1Ab weighed less than the controls. Exposure to environmentally relevant concentrations of eCry3.1 Ab is unlikely to result in risk to the ground beetle: the measured reduction in weight is slight, no effects on survival were observed, and the exposure scenario modelled in the study is extremely conservative. Furthermore, if the protection goal is preservation of predation of soil pests, small decreases in growth are unlikely to have adverse effects, particularly if more than one species provides that function (Raybould et al. 2011).

HQs are generally considered a conservative means to assess risk because the estimates of the NOAECs and EECs are not mitigated by environmental complexity. If HQs for a sufficiently broad range of surrogate species do not exceed a level of concern (here EEC/NOAEC $=1$ ), negligible risk to NTOs from exposure to the insecticidal protein from cultivation of the particular crop may be concluded without further studies (Garcia-Alonso et al. 2006; Romeis et al. 2008). Reviews of field studies of Bt crops on NTOs indicate that conclusions of negligible risk to NTOs based on laboratory effects studies and conservative estimates of exposure are reliable (Romeis et al. 2006; Marvier et al. 2007; Duan et al. 2010); therefore, negligible risk to NTOs from exposure to eCry3.1Ab via cultivation of Event 5307 maize may be concluded with confidence from the laboratory effects data and conservative estimates of exposure based on concentrations of eCry3.1Ab in the crop.

Acknowledgments We thank the many scientists who directed studies summarised in this paper: Mark Bednarcik, Kate Benyon, Catherine Kramer, Natalie Ruddle, and Laura Seastrum at Syngenta; Stephen Vinall, Alexandra McCormac, and Dan Spincer at Mambo-Tox Ltd.; Michael Patnaude,
Christian Picard, and Lee Sayers at Smithers Viscient; Sabine Rentschler at Eurofins Agroscience Services GmbH; and Patrick Hubbard at Wildlife International, Ltd.

Open Access This article is distributed under the terms of the Creative Commons Attribution License which permits any use, distribution, and reproduction in any medium, provided the original author(s) and the source are credited.

\section{References}

ASTM (2004) Guide for conducting acute toxicity tests with fishes, macro invertebrates and amphibians. Standard E729-88. In: Annual book of ASTM standards, vol 11.004, Philadelphia, PA

Bravo A, Soberón M (2008) How to cope with insect resistance to Bt toxins? Trends Biotechnol 26:573-579

Crocker D, Hart A, Gurney J, McCoy C (2002) Project PN0908: methods for estimating daily food intake of wild birds and mammals. Central Science Laboratory, York. Unpublished report to the (UK) Department for Environment, Food and Rural Affairs. http://www.pesticides.gov.uk/Resources/ CRD/Migrated-Resources/Documents/R/Research_PN0908. pdf

Duan JJ, Lundgren JG, Naranjo S, Marvier M (2010) Extrapolating non-target risk of Bt crops from laboratory to field. Biol Lett 6:74-77

Garcia-Alonso M, Jacob E, Raybould A, Nickson TE, Sowig P, Willekens H, Van Der Kouwe P, Layton R, Amijee F, Fuentes AM, Tencalla F (2006) A tiered system for assessing the risk of genetically modified plants to nontarget organisms. Environ Biosaf Res 5:57-65

Grimm C, Reber B, Barth M, Candolfi MP, Drexler A, Maus C, Moreth L, Ufer A (2000) A test for evaluating the chronic effects of plant protection products on the rove beetle Aleochara bilineata Gyll. (Coleoptera: Staphylinidae) under laboratory and extended laboratory test conditions. In: Guidelines to evaluate side-effects of plant protection products to non-target arthropods. Candolfi MP, Blümel S, Forster R, Bakker FM, Grimm C, Hassan SA, Heimbach U, Mead-Briggs MA, Reber B, Schmuck R, Vogt H (eds) International Organization for Biological and Integrated Control of Noxious Animals and Plants, West Paleartic Regional Section (IOBC/WPRS), Gent, pp 1-12

Heimbach U (1992) Testing the effects of plant protection products on larvae of the carabid beetle Poecilus cupreus (Coleoptera: Carabidae). IOBC/WPRS Bull 15:103-109

Jensen PD, Dively GP, Swan CM, Lamp WO (2010) Exposure and nontarget effects of transgenic Bt corn debris in streams. Environ Entomol 39:707-714

Marvier M, McCreedy C, Regetz J, Kareiva P (2007) A metaanalysis of effects of Bt cotton and maize on nontarget invertebrates. Science 316:1475-1477

Nguyen HT, Jehle JA (2009) Expression of Cry3Bb1 in transgenic corn MON88017. J Agric Food Chem 57:9990-9996

NRC (National Research Council) (1983) Nutrient requirements of warmwater fishes and shellfishes. National Academy Press, Washington, DC 
OECD (1984) Guideline for testing of chemicals, no. 207. Earthworm, acute toxicity tests. Adopted 4 Apr 1984

$\operatorname{OECD}$ (2001) Guideline for the testing of chemicals, no. 420. Acute oral toxicity - fixed dose procedure. Adopted 17 Dec 2001

OECD (2007) Series on testing and assessment, no. 75. Guidance document on the honey bee (Apis mellifera L.) brood test under semi-field conditions. Adopted 31 Aug 2007

Oomen PA, DeReuijter A, Van Der Steen J (1992) Method for honeybee brood feeding tests with insect growth-regulating insecticides. EPPO Bull 22:613-616

OPPTS (1996a) Ecological effects test guidelines (draft), no. 850.1020. Gammarid acute toxicity test

OPPTS (1996b) Ecological effects test guidelines (draft), no. 850.2100. Avian acute oral toxicity test

OPPTS (1996c) Microbial pesticide test guidelines, no. 885.4340. Nontarget insect testing, tier I

Palm CJ, Donegan K, Harris D, Seidler RJ (1994) Quantification in soil of Bacillus thuringiensis var. kurstaki $\delta$-endotoxin from transgenic plants. Mol Ecol 3:145-151

Raybould A, Vlachos D (2011) Non-target organism effects tests on Vip3A and their application to the ecological risk assessment for cultivation of MIR162 maize. Transgenic Res 20:599-611

Raybould A, Stacey D, Vlachos D, Graser G, Li X, Joseph R (2007) Non-target organism risk assessment of MIR604 maize expressing mCry $3 \mathrm{~A}$ for control of corn rootworm. J Appl Entomol 131:391-399

Raybould A, Caron-Lormier G, Bohan DA (2011) The derivation and interpretation of hazard quotients to assess the ecological risks from the cultivation of insect-resistant transgenic crops. J Agric Food Chem 59:5877-5885

Raybould A, Kilby P, Graser G (2013) Characterising microbial protein test substances and establishing their equivalence with plant-produced proteins for use in risk assessments of transgenic crops. Transgenic Res 22:445-460

Romeis J, Meissle M, Bigler F (2006) Transgenic crops expressing Bacillus thuringiensis toxins and biological control. Nat Biotechnol 24:63-71

Romeis J, Bartsch D, Bigler F, Candolfi MP, Gielkens MMC, Hartley SE, Hellmich RL, Huesing JE, Jepson PC, Layton R, Quemada H, Raybould A, Rose RI, Sears MK, Shelton J, Sweet J, Vaituzis Z, Wolt JD (2008) Assessment of risk of insect-resistant transgenic crops to nontarget arthropods. Nat Biotechnol 26:203-208

Romeis J, Raybould A, Bigler F, Candolfi MP, Hellmich RL, Huesing J, Shelton A (2013) Deriving criteria to select arthropod species for laboratory tests to assess the ecological risks from cultivating arthropod-resistant transgenic crops. Chemosphere 90:901-909

Vlachos D, Huber S (2011) Petition for determination of nonregulated status for rootworm-resistant Event 5307 corn. http://www.aphis.usda.gov/brs/aphisdocs/10_33601p.pdf

Walters FS, deFontes CM, Hart H, Warren GW, Chen JS (2010) Lepidopteran-active variable-region sequence imparts coleopteran activity in eCry3.1Ab, an engineered Bacillus thuringiensis hybrid insecticidal protein. Appl Environ Microbiol 76:3082-3088 